\title{
0905 IDENTIFYING MODIFIABLE ENVIRONMENTAL RISK FACTORS FOR FALLS IN OLDER PEOPLE
}

S Faulkner*, D Burns Correspondence: National Centre for Prosthetics and Orthotics, University of Strathclyde, Curran Building, 131 St James' Road, University of Strathclyde, Glasgow G4 OLS, UK

10.1136/ip.2010.029215.905

Background With the number of people over the age of 60 growing more than any other population group the issue of falls among the elderly is a growing safety concern. WHO figures show that approximately $28-35 \%$ of those aged $\geq 65$ fall each year with significant socioeconomic and health impacts. The issue of outdoor falls in the elderly is under-researched in comparison to falls in the home.

Methods A systematic review of modifiable outdoor environmental risk factors highlighting the importance of footwear as an interface between the individual and the outdoor environment for the prevention of falls.

Findings Despite the limited amount of time that older people spend outdoors walking is the activity during which the highest proportion of older people falls, citing an uneven surface or tripping as the cause of the fall. Nearly $70 \%$ of active elderly adults report walking as their predominant choice of physical activity. This group have been identified as being at greatest risk of an outdoor fall. When four or more risk factors are identified the potential for a fall significantly increases. It has been suggested that simple effective measures such as appropriate footwear, maintenance of outdoor walking surfaces, improved lighting, additional seating and clearing footpaths frequently of debris and snow may reduce the number of outdoor falls.

Conclusion Identification and reduction of environmental risk factors is thought to reduce the potential for falls. However, this literature review shows that the evidence is inconclusive and highlights the need for further research in this area. 